$20 \mid 1999$

Varia

\title{
La raison des effets, concept polémique
}

\section{Dominique Descotes}

\section{OpenEdition \\ Journals}

Édition électronique

URL : http://journals.openedition.org/ccibp/551

DOI : 10.4000/ccibp.551

ISSN : 2493-7460

\section{Éditeur}

Centre international Blaise Pascal

\section{Édition imprimée}

Date de publication : 19 octobre 1999

Pagination : $39-46$

ISSN : 0249-6674

\section{Référence électronique}

Dominique Descotes, "La raison des effets, concept polémique », Courrier du Centre international Blaise Pascal [En ligne], 20 | 1999, mis en ligne le 06 janvier 2016, consulté le 01 mai 2019. URL : http:// journals.openedition.org/ccibp/551 ; DOI : 10.4000/ccibp.551

Ce document a été généré automatiquement le 1 mai 2019.

Centre international Blaise Pascal 


\title{
La raison des effets, concept polémique
}

\author{
Dominique Descotes
}

1 Le concept de raison des effets, dont au colloque Méthodes chez Pascal de 1976, Yoichi Maeda a souligné l'origine physique, n'est pas polémique par lui-même et n'aurait, selon toute apparence, aucune raison de le devenir : il ne fait, dans son principe, qu'exprimer une certaine conception de l'explication mécanique. Et pourtant, les fragments 124, 126, 127, 132 et $134^{1}$ des Pensées témoignent du fait que les virtualités qui aboutissent à lui donner une portée polémique étaient déjà présentes dans les textes scientifiques de 1647 et 1654, étant entendu que le mot polémique s'entend, non pas au sens de la controverse violente, mais au sens plus modéré de ce qu'Arnauld appelle la réfutation forte, dont Pascal a donné la théorie dans la XI Provinciale, et qui n'enferme aucun procédé blessant ${ }^{2}$.

\section{I - Le concept de raison des effets}

2 Dans la langue de Pascal les effets désignent des phénomènes naturels : non des faits élémentaires bruts recueillis au terme d'une observation conduite au hasard et sans idée préconçue, mais des faits déjà élaborés ${ }^{3}$. Ils apparaissent toujours au travers de la structure complexe d'une expérience, et comme ils sont composés de plusieurs éléments qui paraissent incompatibles entre eux, ils enferment un aspect paradoxal, étonnant, parfois incompréhensible. Ils sont toujours choisis avec cette pensée qu'ils appellent une explication : l'effet est un fait en attente de raison.

3 Les expériences de Pascal sur le vide présentent de tels effets. Elles sont très éloignées de ces expériences simples et ordinaires dont Descartes pense qu'elles suffisent au début de la recherche. Les dispositifs les plus élémentaires en apparence des Expériences nouvelles sont en réalité de conception fort élaborée. Le tube de Torricelli, dès lors qu'il entre en comparaison avec le tuyau de quarante-six pieds, la seringue, le soufflet et le siphon, apparaît comme un cas particulier dans un système complexe de configurations mécaniques : le mercure y laisse apparaître un vide en tombant sous l'effet de son propre poids, par opposition aux expériences effectuées avec de l'eau, à celles où le mercure 
monte dans la seringue, à celles où le tube comporte deux jambes comme le siphon. L'effet y est étroitement défini par tout ce qui n'est pas lui. Les phénomènes que cette expérience révèle suscitent une surprise à effet multiple : la première Expérience nouvelle étonne parce qu'elle montre, contre l'avis des philosophes, qu'une force médiocre suffit pour faire apparaître un espace vide dans le corps d'une seringue, mais aussi parce que cet espace croît sans que la force nécessaire pour l'engendrer ni le poids de la seringue ne varient. D'ailleurs, dans l'expérience de Torricelli, on comprendrait à la rigueur que le mercure demeure suspendu de manière à remplir complètement le tube barométrique, ou au contraire qu'il l'évacue pour tomber tout entier dans la cuve ; mais pourquoi demeuret-il suspendu à une hauteur intermédiaire? Le plus souvent, la surprise naît d'une disproportion: c'est l'essence du paradoxe hydrostatique, que le P. Mersenne exprime en notant qu'un filet d'eau tient en équilibre l'océan, ou de la presse hydraulique, que Pascal décrit en ces termes: «Si un vaisseau plein d'eau, clos de toutes parts, a deux ouvertures, l'une centuple de l'autre; en mettant à chacune un piston qui lui soit juste, un homme poussant le plus petit piston égalera la force de cent hommes qui pousseront celui qui est cent fois plus large, et en surmontera quatre-vingt-dix-neuf ${ }^{4}$. » Dans les Pensées, la même disproportion dans les phénomènes apparaît lorsque la pensée d'un « souverain juge du monde » est empêchée par une mouche qui bourdonne, dans le fait que «les Suisses s'offensent d'être dits gentilshommes ", ou que le hasard dispose du choix du métier, alors que c'est la chose la plus importante à toute la vie.

4 Tout autant qu'effet, le mot raison demande explication. Dans ses traités de physique, Pascal parle aussi bien de cause que de raison des effets. Le chapitre II de L'Équilibre des Liqueurs indique qu'il faut prendre " pour très véritable qu'un vaisseau plein d'eau, ayant des ouvertures, et des forces à ces ouvertures qui leur soient proportionnées, elles sont en équilibre » : et c'est le fondement et la raison de l'équilibre des liqueurs. Pascal écrit aussi à propos de la machine hydraulique: "l'on doit admirer qu'il se rencontre en cette machine nouvelle cet ordre constant qui se trouve en toutes les anciennes, savoir le levier, le tour, la vis sans fin, etc., qui est que le chemin est augmenté en même proportion que la force (...) ; de sorte que le chemin est au chemin comme la force à la force. Ce que l'on peut prendre même pour la vraie cause de cet effet, étant clair que c'est la même chose de faire faire cent pouces de chemin à une livre d'eau ».

Cause désigne l'élément efficace qui provoque un phénomène, mais n'enferme pas l'idée d'explication: on peut constater une cause sans comprendre comment elle opère, par exemple savoir que sur une balance, un poids médiocre peut en contrepeser un plus grand, sans connaître la loi mécanique qui explique cet équilibre. Raison insiste au contraire sur le fait que l'on comprend comment et pourquoi une certaine configuration de forces engendre un phénomène. Le passage de L'Équilibre des liqueurs cité plus haut est particulièrement instructif sur ce point, car la règle, qui exprime la raison des effets, « c'est la même chose de faire faire un pouce de chemin à cent livres d'eau ", n'est qu'une autre formulation de celle qu'à énoncée Stevin: l'espace parcouru par la puissance est à l'espace parcouru par la résistance comme cette résistance est à la puissance :

Comme l'espace de l'agent, à l'espace du patient,

Ainsi la puissance du patient, à la puissance de l'agent ${ }^{5}$.

C'est au sens littéral une proposition, c'est-à-dire une égalité de raisons ${ }^{6}$. Dans raison des effets, raison doit, en son origine au moins, être pris au sens mathématique de proportion, et désigne une loi abstraite qui régit les rapports entre éléments qui composent les effets. C'est en ce sens qu'elle supprime l'étonnement et rétablit l'intelligence: elle réintroduit 
la proportion là où la seule observation des phénomènes ne montrait qu'une disproportion. De même, lorsque que l'on sait, comme l'écrit Dettonville, «que les forces des bras sont en raison composées des poids et des bras ", on rétablit la proportion par l'égalité du produit des poids par les distances au centre de gravité, ce qui supprime le paradoxe de l'équilibre des poids inégaux sur la balance. Enfin, la règle qui énonce que les liqueurs pèsent suivant leur hauteur donne aussi la raison des effets de l'équilibre de l'eau dans les vases communicants et de son mouvement dans les siphons. C'est à partir de là que la notion de cause prend son sens : la proportion, qui définit l'homogénéité, est la condition d'une relation causale : « quae sunt heterogenea quomodo inter se adfecta sint, cognosci non potest ", écrit Viète citant Adraste ${ }^{7}$. L'homogénéité rétablie, l'existence d'une liaison de causalité va d'elle-même. C'est proprement ce qui distingue la mécanique moderne de l'ancienne: la physique scolastique s'inquiète des causes, sans chercher la proportion ; la mécanique moderne recherche les rapports qui proportionnent les causes et leurs effets.

7 C'est ainsi par exemple que, dans La pesanteur de la masse de l'air, Pascal explique le mouvement du mercure dans un siphon, par le calcul de la proportion des forces exercées par les deux colonnes de liquides. Dans la cuve remplie d'eau, le mercure contenu dans le vaisseau inférieur subit une pression dont la force est égale à celle d'une colonne d'eau de hauteur $(X+1$ pouce), plus grande d'un pouce que la colonne de hauteur $X$ qui pèse sur le vaisseau supérieur. Quoique l'eau pèse sur le vaisseau inférieur plus que l'autre « de la force que lui donne la hauteur d'un pouce », il ne faut pas conclure que le mercure ira s'élevant du vaisseau inférieur vers le supérieur; il faut comparer les forces exercées par le mercure et l'eau: si l'eau presse le vaisseau inférieur de la force que lui donne un pouce de hauteur, le mercure de la jambe pèse de la force que lui donne un pouce de mercure, qui est évidemment plus grande : ce qui explique que le mouvement du mercure va de la courte jambe à la plus longue.

8 On comprend pourquoi Pascal écrit que les effets sont visibles, mais que leur raison ne l'est pas. Une proportion mathématique n'est pas l'objet d'une intuition visuelle, elle n'est concevable que par l'esprit, d'autant plus qu'elle enferme une construction intellectuelle : le produit de la force par l'espace, qui définit le travail, est une grandeur abstraite, par nature inaccessible aux sens.

Schéma représentant le mouvement du mercure dans le siphon

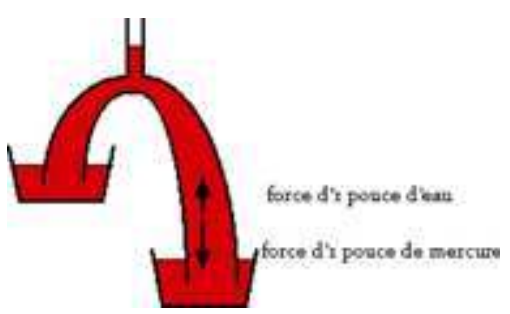

Réalisé par Dominique Descotes à partir de La pesanteur de la masse de l'air

9 C'est l'office des machines de la rendre perceptible à l'imagination rationnelle. Ainsi, dans L'Équilibre des liqueurs, Pascal remarque que la presse hydraulique, «cette machine de mécanique pour multiplier les forces", fait «voir la raison pour laquelle les liqueurs pèsent suivants leurs hauteur »: les phénomènes s'y conforment si exactement à la règle de proportion des espaces et des forces qu'elle en devient pour ainsi dire l'emblème. 
Schéma représentant le mouvement du mercure dans le siphon dans une cuve remplie d'eau

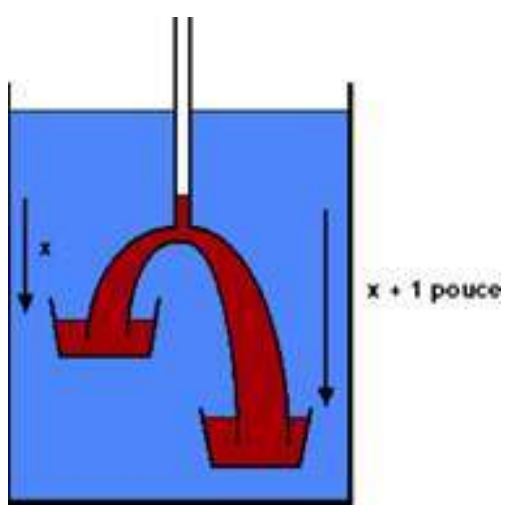

Réalisé par Dominique Descotes à partir de La pesanteur de la masse de l'air

\section{II - Le tournant polémique}

Dans tout cela, rien qui semble relever de la polémique. Le germe est pourtant présent dès les textes de physique de 1647.

11 La technique de Pascal consiste à mettre en scène les individus qui se trompent au lieu de se contenter de réfuter les erreurs, à faire de la compréhension et de l'incompréhension de la raison des effets le thème même de son argumentation. Il associe la connaissance des effets et de leur raison à différents point de vue, caractérisés chacun par une perspective, des principes, qui déterminent sa mentalité, sa manière de penser particulière. La distinction des opinions se prolonge ainsi en une distinction des types d'esprits, construite sur le modèle visuel, selon ce que les esprits voient ou ne voient pas, et la manière dont ils le voient, selon que la vue bornée s'arrête aux effets, ou au contraire qu'elle en comprend la proportion cachée, les causes et leur mode d'action dans les phénomènes ${ }^{8}$. De ce fait, comme les degrés de la gradation possèdent chacun des principes différents, la communication entre eux est très difficile, parfois pratiquement impossible. Le demi-habile par exemple voit " de toute sa vue » les grands de naissance comme des êtres ordinaires, alors que les naïfs voient en eux des êtres supérieurs : avec des principes si différents, quel terrain commun pourraient-ils trouver? Ils sont dans la situation du fragment 132 : un esprit boiteux irrite, mais comme il « dit que c'est nous qui boitons ", on voit mal comme on pourrait le convaincre qu'il a tort. En effet, le demihabile ne discute pas avec l'homme du peuple; il le méprise pour sa naïveté. Entre l'habile et le demi-habile, il y a quelques principes communs, mais la discussion risque aussi de tourner au dialogue de sourds. Pour désigner les rapports de contrariété qui opposent ces différents points de vue, Pascal use d'un verbe particulier : on ne réfute pas les opinions du peuple ou des demi-habiles : on les détruit, autrement dit on les disqualifie, on les rend nulles et non avenues, ridicules ou méprisables, à partir des principes d'ordre supérieur.

Des types d'esprits aux personnes, la transition est effectuée par le truchement de figures emblématiques : dans les Pensées, Montaigne, Épictète, saint Augustin même, sont de ceux dont le regard est borné aux effets : ils représentent par délégation dans le texte certaines personnes plus actuelles, caractérisées par une mentalité analogue. Le malheur du Père Noël dans sa polémique avec Pascal, c'est d'avoir été réduit à tenir lieu d'une telle figure 
emblématique, celle du demi-habile en physique. Cette technique transforme le modèle de la raison des effets en argument ad hominem 9 .

13 Le peuple compose une première catégorie, celle qui ne voit que des phénomènes simples : il ne distingue pas l'essence et l'apparence, la loi établie et la justice essentielle ; à ses yeux la grandeur d'établissement coïncide avec la grandeur naturelle ; à ses yeux un prince est nécessairement un être de nature supérieure. Dans la physique, le bas de l'échelle est tenu par les artisans, qui constatent les faits sans pouvoir en donner une interprétation rationnelle. Chez les uns et les autres, on retrouve le même dogmatisme naîf : la confiance que le peuple accorde aux lois de son pays vaut l'assurance des fontainiers qui croient pouvoir élever l'eau jusqu'à n'importe quelle hauteur.

politique et en morale, le demi-habile, lui, discerne l'essence et l'apparence que le peuple confond. Il sait que la loi n'est pas essentiellement juste parce qu'elle est établie ; il est donc en mesure de remarquer des effets, au sens pascalien du mot, c'est-à-dire les anomalies qui échappent au peuple, par exemple la disproportion qui existe entre la grandeur d'établissement d'un prince et sa valeur personnelle. On notera que Pascal classe implicitement les philosophes parmi les demi-habiles : qu'il s'agisse des sceptiques comme Montaigne ou des Stoïciens comme Épictète, tous ont en commun d'avoir vu que la nature humaine est pleine de paradoxes, et d'avoir construit de grands systèmes pour en rendre compte, faute d'avoir connu la raison des effets, c'est-à-dire la doctrine du péché originel. Il existe aussi des demi-habiles en géométrie, comme l'indiquent les Lettres de A. Dettonville $e^{10}$ : ce sont ceux qui s'imaginent que c'est pécher contre la géométrie que d'user des indivisibles. Leur point de départ est juste en un sens: ils pensent, conformément aux principes fondamentaux de la géométrie, que l'on ne doit pas mélanger les genres, et que l'on n'engendre pas des lignes à partir des points, ni des surfaces en ajoutant des lignes les unes aux autres. C'est précisément ce que dit Pascal dans L'esprit géométrique. Mais si cette règle fondamentale d'homogénéité est indispensable pour prendre rang parmi les géomètres, elle ne suffit pas pour faire un géomètre habile : appliquée sans discernement, elle se transforme en œillère. Pascal s'en explique dans les Avertissements de la Lettre à Carcavy: ceux qui récusent les indivisibles sous prétexte qu'on n'a pas le droit d'exprimer une surface par une somme de lignes, manquent d'intelligence, c'est-à-dire qu'ils n'ont pas compris la convention qui fonde la méthode : lorsque l'on parle de somme de lignes, on entend que ces lignes sont multipliées par des portions infiniment petites qui en font des rectangles, si bien que la règle d'homogénéité n'est pas violée. Il en résulte que par myopie intellectuelle, un géomètre demi-habile comme le $\mathrm{P}$. Lalouvère se prive d'une méthode féconde.

Le cas de la physique est plus intéressant, car les demi-habiles s'y présentent dans toute leur variété. Ce sont d'abord les purs philosophes scolastiques comme Pierius, mais aussi ceux qui, comme le P. Noël, greffent un peu de cartésianisme sur Aristote. Ils ont en commun de ne pas comprendre comment une force médiocre peut être capable d'engendrer le vide dans une seringue. C'est un mérite incontestable de s'en étonner, et c'est en cela qu'ils sont habiles; mais leur tort, c'est d'inventer des causes imaginaires pour ne pas dévoiler leur ignorance: une matière invisible qui emplit l'espace laissé vacant, un mouvement circulaire de particules analogue à l'antipéristase des anciens, la légèreté mouvante. Tout cela vaut bien les constructions morales et métaphysiques des philosophes stoïciens ou cartésiens pour rendre compte de la nature humaine. Mais il existe aussi des esprits plus instruits : ce sont les partisans de l'horreur mesurée du vide contre lesquels Pascal réalise la Grande Expérience du Puy-de-Dôme, tel Galilée, dont 
l'hypothèse de la Altessa limitatissima ne correspond qu'à une demi-compréhension de la mécanique des liquides ; mais tout erronée qu'elle soit, cette opinion n'en représente pas moins un progrès par rapport à d'autres, puisqu'elle suppose assimilée l'idée de la pesanteur de l'air.

Au-dessus se trouvent les habiles, qui connaissent la raison des effets. Naturellement, c'est en général la position que, dans les textes, occupe le personnage de l'auteur. En géométrie, l'image en est donnée par Amos Dettonville, honnête homme capable tout à la fois de découvrir des propriétés nouvelles, et d'expliquer les fondements de la méthode qui a permis de les démontrer. Il semble que, dans le projet d'Apologie, le rôle de l'habile homme qui connaît la raison des effets devait être tenu par le personnage de l'auteur, sous le nom de Salomon de Tultie. Dans les Pensées, l'habile s'apparente au demi-habile par la connaissance qu'il a de la différence entre justice essentielle et loi établie, mais il ressemble au peuple par le fait que la pensée de derrière lui fait refuser de contester publiquement l'ordre établi ${ }^{11}$.

Pascal ajoute deux degrés, les dévots et les chrétiens parfaits. Ils se situent au-dessus des précédents par le fait qu'ils jouissent d'une source de lumière supplémentaire, par la foi chrétienne. Mais pour le reste, ils ressemblent, pour les dévots, aux demi-habiles ; pour les chrétiens parfaits, aux habiles. Ni la physique ni la géométrie ne comportent d'équivalent. Aussi reviendrons-nous plus bas sur ces deux catégories.

Les arguments polémiques composés par Pascal dépendent des différents aspects de cette gradation. Une première série d'arguments correspond au fait que ces degrés représentent des points de vue dont les axiomatiques sont hétérogènes, et ordonnés selon une gradation croissante. Enfin, une deuxième catégorie, propre aux seules Pensées, et qui n'a pas d'équivalent dans les autres textes, répond au fait que, malgré leur hétérogénéité, les différents points de vue présentent des ressemblances qui permettent de les réduire les uns aux autres.

\section{Première série : arguments de contradiction}

19 Certains arguments sont relatifs à la matière : ils consistent à montrer que certaines doctrines ne tiennent pas compte de toutes les données, parce que les points de vue correspondants sont partiels et incomplets. Ils jouent donc sur l'écart et la gradation qui existe entre les différents points de vue. La restriction de l'information sert ainsi à discréditer certains degrés. Lorsque Pascal veut, dans la Conclusion de ses traités de 1654, montrer en quoi les nouvelles doctrines sont supérieures aux anciennes, il commence par souligner que, du point de vue de l'expérience, les anciens n'avaient sur le vide que des connaissances très restreintes : ils n'ont pas cherché des épreuves bien loin, ni construit des instruments d'assez grande dimension pour voir si le vide était possible. De même, si le peuple croit que les lois locales expriment la vraie justice, c'est qu'il n'est jamais sorti de chez lui pour expérimenter que les lois ne sont pas les mêmes en deçà et au-delà des Pyrénées. L'usage polémique de la raison des effets apparait alors dans la manière dont Pascal choisit les effets de manière à montrer les insuffisances de ces points de vue. Cette disposition d'esprit apparaît dès les premières recherches sur le vide, lorsque Pascal reproduit l'expérience d'Italie avec Petit et son Père : il demande ce qu'il faudra répondre aux simpliciens ; autant que sur les phénomènes observés, sa réflexion porte sur ceux qui se trompent. Les Expériences nouvelles sont explicitement construites de manière à montrer que les phénomènes vont «contre le sentiment des philosophes » : s'étant fait 
une idée des conséquences que leurs principes comportent, il construit ses expériences de manière à produire justement les phénomènes qu'ils déclarent impossibles « avec aucune force finie ». L'expérience du Puy-de-Dôme est conçue non pas seulement pour établir définitivement que la pression de l'air est la cause de la suspension du mercure dans le tube barométrique, mais aussi pour en finir avec la thèse de l'horreur du vide.

Le développement de cet argument aboutit à la réduction à l'absurde. Qu'on laisse les demi-habiles raisonner selon leurs principes, on aboutit tout droit à des conséquences si absurdes qu'ils les désavoueront eux-mêmes si l'évidence de faits ne les prévient pas. Imaginons en effet, comme en géométrie, un demi-habile qui aurait assimilé la règle d'équilibre des liqueurs, un peu comme certains géomètres ont assimilé la règle des hétérogènes en géométrie. On le verra, de la même manière, appliquer cette règle à tort et à travers. Pascal le démontre dans La pesanteur de la masse de l'air à propos de l'expérience du siphon décrite ci-dessus. Partant du principe que les liqueurs pèsent selon leur hauteur, et constatant que la colonne d'eau qui pèse sur la cuve la plus basse est plus grande que l'autre, le demi-habile conclura avec la meilleure volonté du monde que le vifargent doit être poussé de la jambe la plus longue dans la plus courte, et remonter dans le siphon. C'est évidemment l'inverse qui se produit. La Conclusion des traités propose un exemple encore plus satirique, lorsque Pascal conclut qu'en raisonnant comme les tenants de l'horreur du vide, on devrait logiquement conclure qu'un siphon plongé dans l'eau, dont l'extrémité ouverte atteint l'air libre, est difficile à ouvrir parce qu'il a horreur de l'air.

21 D'un genre proche est l'argument d'inutilité: le demi-habile croit que le mélange des ordres est propre à résoudre certaines difficultés, mais ce n'est qu'une apparence. En politique, il est inutile de prétendre ordonner la grandeur d'établissement qu'est le titre de prince sur la grandeur spirituelle qu'est son mérite personnel, ou de vouloir ordonner les lois établies sur une justice essentielle dont l'homme n'a qu'une connaissance obscure, tout cela est voué à l'échec. Cet argument a l'intérêt de dévoiler que la demi-habileté est toujours une sorte de tyrannie. La XII ${ }^{e}$ Provinciale le proclame avec éloquence : ce sont les demi-habiles qui croient pouvoir étouffer la vérité par la force; mais cet effort demeure sans effet, puisque la violence et la vérité ne peuvent rien l'une sur l'autre. L'argument d'impossibilité en est une variante : parmi les philosophes, c'est aussi une forme de demihabileté de croire qu'il est en notre pouvoir de régler le cœur.

Un pas de plus, et l'on tombe dans l'argument par contradiction, qui montre que non seulement les demi-habiles veulent réaliser l'impossible, mais qu'ils aboutissent au résultat directement contraire à celui qu'ils recherchent: c'est celui par lequel Pascal soutient que les principes des demi-habiles conduisent tout droit à la guerre civile. Que l'on récuse toutes les lois en vigueur, que l'on en appelle à la loi naturelle et aux lois fondamentales du royaume, que l'on enlève au peuple l'estime qu'il a des lois en vigueur et des principes qui le gouvernent, et c'est la fronde, c'est-à-dire l'exact contraire de ce que l'on cherchait.

Ces arguments ne peuvent être disjoints de ceux qui jouent sur la gradation des types au sein d'une hiérarchie. Certains points de vue sont supérieurs aux autres, parce que, de degré en degré, la perspective s'élève, de sorte que chacun voit plus loin ou mieux que le précédent, ou en termes géométriques, que son axiomatique est plus complexe. Il en résulte que c'est toujours d'un point de vue supérieur que s'effectue la critique d'un point de vue inférieur. Dans les Pensées, le demi-habile, quoique supérieur au peuple, est inférieur à l'habile parce qu'il ne tient pas compte des exigences fondamentales que 
l'habit connaît: les mesures qu'il prône conduisent infailliblement à la guerre civile, parce qu'il lui manque le sens du réel, du possible et du nécessaire, qualités qui sont précisément celles de l'habile. Ce dernier a intégré à son calcul la nécessité fondamentale de la sécurité, et c'est au nom de l'intérêt général bien compris qu'il préfère l'esprit de réforme à l'esprit de fronde. Il en va de même pour les dévots et les parfaits chrétiens. Si un mépris réciproque oppose les différents degrés, les termes n'en sont pas pour autant symétriques. Ces oppositions sont liées à des rapports de supériorité et d'infériorité, qui font que chaque degré supérieur comprend le mécanisme de la pensée des degrés inférieurs, sans être vraiment ni compris ni jugé par eux. Du fait qu'ils distinguent ce que le peuple confond, les demi-habiles comprennent le peuple mieux que le peuple ne se comprend lui-même ; ils méprisent sa naïveté, sa sottise, et la facilité avec laquelle les puissants le dupent et l'asservissent. Mais cette relation est asymétrique : le peuple, lui ne comprend pas la pensée des demi-habiles. De la même manière, les habiles comprennent l'erreur des demi-habiles, mais la réciproque n'est pas vraie, car le demi-habile ignore la pensée de derrière. Quant aux habiles, ils ne comprendront jamais les dévots, ni les parfaits chrétiens.

24 Ainsi la destruction des opinions s'effectue selon le modèle du renversement du pour au contre, selon le modèle du fragment 127, en progressant d'une opinion à l'autre, du bas de l'échelle au sommet. Chaque opinion contredit la précédente, au nom de principes qui ne sont pas les siens, mais qui sont plus compréhensifs, de telle manière que chaque degré comprend le précédent sans être compris par lui.

\section{Deuxième série : arguments de réduction}

25 Quoique les degrés successifs de la gradation différent par leurs principes, ce qui fait l'originalité de la gradation dans les Pensées, c'est que Pascal y a ajouté des concordances entre les différents types qui la constituent, qui permettent de compliquer les argumentations par des procédés de court-circuit particulièrement efficaces dans la polémique.

Quoique les parfaits chrétiens en sachent plus que les habiles et les habiles que le peuple, ils ont pourtant un point commun : tous sont d'accord pour honorer les gens de grande naissance et pour éviter de troubler la société par des frondes. Quoique les dévots en sachent plus que les demi-habiles, ils ont ceci de commun qu'ils refusent d'honorer les gens de grande naissance et qu'ils sont, chacun à leur manière, des trublions et des frondeurs. Ainsi, malgré les différences qui les séparent dans les principes, les conclusions et les positions pratiques coïncidentes : un parfait chrétien, un habile et un homme du peuple se conduisent à peu près de la même manière, et l'on peut dire que, même si les opinions du peuple sont "très fausses ", elles sont aussi "saines " dans la mesure où elles rencontrent celles de la plus grande habileté. Cette coïncidence régulière des termes pairs et impairs de la gradation est absente des écrits de physique, où les degrés sont tous différents les uns des autres : ni les thèses ni les principes ne sont les mêmes ; il n'y a pas de ressemblance entre les artisans, premier degré de la gradation, et les vrais savants. C'est même un argument dont Pascal use en faveur du vide, de dire que ses ennemis sont tous d'avis différents, sans rien de commun.

Si l'on convient de distinguer, dans la gradation, les degrés impairs (le peuple, les habiles, les chrétiens parfaits) et les pairs (les demi-habiles et les dévots), on peut distinguer deux catégories d'arguments. 
28 La première joue sur les coïncidences entre degrés du même ordre, montre les ressemblances entre les termes pairs, ou entre les degrés impairs. Cet argument polémique consiste à relever les degrés inférieurs en raison de leur parenté avec les degrés supérieurs de même ordre, en court-circuitant les degrés intermédiaires. Considérons les impairs: Pascal souligne que les opinions du peuple coïncident avec celles des habiles, et mieux encore avec celles des parfaits chrétiens: le peuple s'en trouve valorisé, et même si ses opinions sont fausses, Pascal conclut qu'elles sont saines. Il faut bien voir qu'il n'y a pas là une coïncidence purement accidentelle, sans effet sur le fond des choses : la santé des opinions du peuple est significative, car elle correspond à la vérité de la condition humaine : le monde juge bien des choses, car il est dans l'ignorance naturelle qui est le vrai siège de l'homme.

29 L'argument peut aussi jouer en sens inverse : le rapprochement des dévots et des demihabiles n'est pas fait pour les rendre considérables : il revient à souligner que le dévot est au fond un brave homme de chrétien, plein de bons sentiments, mais surtout un excité qui n'a pas bien compris ce que Dieu exige de lui.

30 La seconde catégorie, plus subtile, consiste à montrer les parentés entre termes de rang différent, c'est-à-dire entre pairs et impairs. Ce branchement du degré intermédiaire sur le degré inférieur, qui associe les contraires, produit nécessairement un effet de surprise. Il consiste à suggérer que les demi-habiles sont des naîfs qui s'ignorent. On sait que le propre du peuple est de croire que les lois de son pays valent pour l'univers entier : c'est une manière de confondre une justice qu'on imagine avec la justice essentielle. L'argument de Pascal consiste à souligner que les demi-habiles sont au fond de la même farine : ils imaginent un ordre de choses où règne la vraie justice, mais c'est une justice formée selon leur fantaisie, qu'ils ont la prétention funeste d'imposer à tous. D'où il résulte que le demi-habile est un naïf qui s'ignore et que, s'il se jugeait lui-même avec la même sévérité qu'il applique au peuple, il devrait se mépriser.

31 Pascal esquisse différentes formes de cet argument qui réduit le demi-habile à des manières de penser prosaïquement raisonnables et populaires. Citons, dans ce registre, l'argument de la poule de Tertullien : contre l'objection des incrédules qui consiste à dire «qu'on ne peut ressusciter», Pascal répond qu'elle n'est qu'une affaire de " manque de coutume » : " populaire façon de juger », chez des gens qui méprisent pourtant le peuple. Autre argument du même genre, celui du fragment 257, selon lequel les «incrédules (sont) les plus crédules ", parce qu'ils n'admettent pas les miracles du Christ, mais acceptent ceux de Vespasien ${ }^{12}$. G. K. Chesterton tirera un parti brillant de ce procédé, dans le premier chapitre du Nommé Jeudi.

Mais il faut considérer que l'argument peut aussi jouer en sens inverse dans une certaine mesure : sous la critique de la bêtise des demi-habiles, il y a aussi un plaidoyer en leur faveur. Dans demi-habile, il y a demi, mais il y a aussi une part d'habileté : à côté des demihabiles sceptiques de profession, «académistes écoliers" insupportables, «le plus méchant caractère d'homme que je connaisse ", il y a aussi le demi-habile Montaigne, qui, lui, n'est pas méprisable. L'Entretien avec M. de Sacy montre, sur ce point, Pascal pense, comme le Claudel des Conversations dans le Loir et Cher, que le demi-habile a aussi son utilité, comme poil à gratter et démangeaison nécessaire qui interdit de se tenir en repos dans l'égoïsme. 


\section{III - Les effets à retardement de la raison des effets} fragment 256 formule en ces termes le programme argumentatif de Pascal: «il faut mettre dans Fondements ce qui est en celui des Figuratifs touchant la cause des figures. Pourquoi Jésus-Christ prophétisé en son premier avènement, pourquoi prophétisé obscurément en la manière ». Il est en effet nécessaire de justifier l'obscurité des prophéties contre la thèse des libre-penseurs, qui ne voient dans les prophètes que des sots, des ignares, ou, comme l'écrira Spinoza, des personnes douées surtout d'une ample imagination, en tout cas des auteurs dépourvus de sagesse philosophique.

37 La réponse de Pascal rappelle directement Raison des effets : elle consiste à soutenir que "Moïse était habile homme», et David "grand témoin» et "bon esprit», que les prophètes en général avaient « assurément trop de bon sens » pour laisser échapper des absurdités; et qu'au contraire les objections contre l'interprétation spirituelle des prophéties ne peuvent provenir que de demi-habiles. Premier argument: reprocher à la religion, et particulièrement aux prophéties leur manque de clarté, c'est ne pas avoir su prendre en compte le fait que l'obscurité est voulue et déclarée par leurs auteurs eux- 
mêmes : « qu'on ne nous reproche donc plus le manque de clarté, puisque nous en faisons profession ». D'autre part, reprocher aux prophètes leur manque de clarté, c'est la preuve qu'on n'en a pas vu la raison nécessaire : si les prophètes ont parlé du Messie en termes figuratifs et obscurs, c'est qu'ils y étaient obligés par la nature spirituelle du Christ. Le fragment sur les trois ordres l'indique expressément : on ne peut parler de la grandeur de Dieu qu'en termes tirés de l'ordre des corps, par exemple des batailles de Dieu et de ses saints contre le péché, qu'avec des images empruntées à l'art de la guerre. Les objections des incrédules sont faites par des demi-habiles qui n'ont pas pris en compte toutes les données du problème. L'argument porte d'ailleurs aussi dans une certaine mesure contre les Juifs. C'est toute la théorie pascalienne de l'interprétation qui est marquée par la problématique de Raison des effets.

Ces directions demanderaient à être explorées dans un plus grand détail que ne le permet le cadre de la présente étude. Elle suffit cependant à donner une idée de la manière dont un usage polémique peut animer un concept, qui, à l'origine, n'était destiné qu'à un emploi d'ordre scientifique. Le génie synthétique de Pascal est plus que tout autre propre à susciter de telles métamorphoses.

\section{NOTES}

1. Les références aux fragments des Pensées sont données dans l'édition de Philippe Sellier.

2. Voir Dominique Descotes, "Force et violence dans le discours chez Antoine Arnauld", in Antoine Arnauld. Philosophie de la connaissance, Études réunies par J-C Pariente, Vrin, Paris, 1995, p. 33-64 ; «De la XI ${ }^{\mathrm{e}}$ Provinciale aux Pensées », in Courrier du Centre International Blaise Pascal, $\mathrm{n}^{\circ} 16$, 1994, p. 35-38.

3. Je renvoie une fois pour toutes à la mise au point de Laurent Thirouin dans le présent Courrier.

4. $O$ C II, p. 1045.

5. Stevin, p. 509 B.

6. J'aurais donc tendance à nuancer un peu la formule de Laurent Thirouin, selon laquelle « la raison est une notion non scientifique ». Mais la divergence n'est qu'apparente.

7. Viete, Isagoge, cap. III, in Opera, p. 2.

8. Voir là-dessus Jean Mesnard, « Point de vue et perspective dans les Pensées de Pascal », Courrier du Centre International Blaise Pascal, 16, 1994, p. 6-7.

9. J'entends argumentation ad hominem au sens de C. Perelman dans le Traité de l'argumentation, I, p. 150, par opposition à l'argument ad personam, attaque contre la personne de l'adversaire destinée à le disqualifier, qui serait une forme de violence rhétorique. Pascal précise bien dans la $\mathrm{XI}^{\mathrm{e}}$ Provinciale les réserves qu'il fait sur l'argument ad personam, en posant la règle de discrétion que doit respecter la polémique chrétienne.

10. OC IV, p. 424.

11. Cette classification s'inspire peut-être dans ses origines de sources libertines: selon le $P$. Garasse, les libertins distinguent, les esprits bas de la populace, les esprits communs, et les esprits écartés, nobles et transcendants (les libertins eux-mêmes). Charron propose une pareille gradation, avec, au bas de l'échelle, les esprits faibles nés pour être menés, les gens médiocre jugement, «qui font profession de suffisance, science, habileté, mais qui ne se sentent pas et ne 
se jugent pas assez, s'arrêtent à ce que l'on tient communément et l'on leur baille du premier coup, sans davantage s'enquérir de la vérité et source des choses » et "pensent que ce que l'on croit en leur village est la vraie touche de vérité », et enfin les hommes " doués d'un esprit vif et clair, jugement fort, ferme et solide, qui ne se contente pas d'un ouï-dire, ne s'arrêtent pas aux opinions communes et reçues": «ceux-ci sont en petit nombre de l'eschole et ressort de Socrates et Platon, modestes, sobres, retenus ».

12. Sel .444, voir l'édition des Pensées de Havet, t. 2, p. 89 ; Sel. 257 ; saint Augustin, Cité de Dieu, I, XIV, t. 33, p. 239 sq. ; Montaigne, De l'art de conférer ; Tacite, Histoires, IV, 81 ; c'est la mise en forme d'un argument de Grotius, De Veritate Religionis christianae, IV, 8.

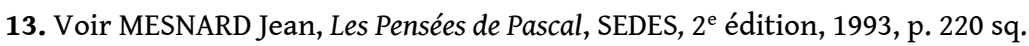

\section{INDEX}

Mots-clés : Pascal, raison des effets, effet, raison, argumentation

Keywords : Pascal, reason, effect, argumentation

\section{AUTEUR}

DOMINIQUE DESCOTES

CERHAC, Université Blaise Pascal 